\title{
How Questions and Answers Shape Online Marketplaces: The Case of Amazon Answer
}

\author{
Warut Khern-am-nuai \\ McGill University \\ warut.khern-am-nuai@mcgill.ca
}

\author{
Hossein Ghasemkhani \\ Purdue University \\ hossein@purdue.edu
}

\author{
Karthik Kannan \\ Purdue University \\ kkarthik@purdue.edu
}

\begin{abstract}
This paper uses data from two online shopping platforms to investigate the economic implications of the Q\&A system. This research problem becomes increasingly important as many websites start to adopt the Q\&A system. Yet, its economic implications have not been discussed in the previous literature. We employ the difference-in-differences analysis to examine the effect of $Q \& A$ elements on product sales. We find that question elements negatively affect sales while answer elements have a positive impact. Also, an increase in the number of question is positively correlated with the number of reviews. Meanwhile, an increase in the number of answers reduces the average length of reviews. Our findings suggest that incorporating the $Q \& A$ system could be a potential approach to drive sales. However, it is crucially important for managers to develop appropriate policies to gather necessary answers to questions asked on the platform in order to capitalize on such system.
\end{abstract}

\section{Introduction}

One of the major obstacles online marketplaces face is the lack of physical touch. This problem is consistently cited as a major roadblock compared to their brick-and-mortar counterparts and usually leads to a trust issue in the eyes of consumers [1]. Several avenues have been proposed by previous literature to alleviate this issue $[2,3]$. One approach that has become increasingly popular in recent years is to integrate a discussion system into the product page. One example of such a system is "Amazon Answer", which was introduced in 2012. This Q\&A system allows any Amazon users to ask and answer questions that are available on Amazon. One question can be answered by more than one users and participants can ask and answer questions without purchasing the product. This paper studies the economic implications of such a system, particularly how the question and answer elements affect product sales.

Although our study is not the first to investigate facilitation of discussions among consumers, there are two main differences in the focus of this paper from previous works. First, the use of online discussions in previous studies is typically in a selling-neutral setting $[4,5]$. There is a limited number of previous works that investigate the economic implications of online discussion platforms. Second, in our context, the online discussion platform co-exists with the review platform, which has been shown to have a significant economic impact on its own $[6,7]$. With the limited insights regarding the economic implication of a Q\&A system from the literature, managers are left with their own intuition to derive relative policies and managerial practices to manage such a system. This lack of guideline is particularly problematic as many small and medium websites have started to adopt the Q\&A system. Furthermore, online discussion and online reviews may share similarities as both of them are typically perceived to be a place for users to share their knowledge. However, their theoretical foundations are drastically different. For one, online review systems typically provide a one-way communication channel where contributors share their experiences, while online discussion systems are built upon two-way interactions between participants who ask and answer questions. In addition, the content shared on both systems are typically different. Online reviews normally contain more generic content, with some degree of experience and opinions. On the other hand, questions and answers tend to be more specific, regarding functions or features of the product, and do not involve much of personal taste or experience. Furthermore, although both online reviews and online discussions could be considered forms of electronic word of mouth, such notion is only well established for online reviews. Therefore, even though online reviews are widely discussed in the previous literature, the implications of an online discussion system is unclear.

We collected the data for the study from Amazon.com and another major online retailer between March and September 2015. While Amazon offers both review and Q\&A system, the other store only offers the standard online review system. With this structure, 
we match products on both platforms and employ the difference-in-differences analysis to unveil the impact of Q\&A elements on product sales. We find that question and answer elements have a significant impact on product sales. Controlling for all other factors, question elements appear to negatively affect sales, especially for popular products. Meanwhile, answer elements seem to affect product sales in a positive manner. Interestingly, although the number of questions hurts product sales, the fraction of questions that have at least one answer has a positive impact on sales. Furthermore, once we focus the analysis on only initial sales, it turns out the the number of questions and the fraction of questions with at least one answer both positively impact sales. We also find that an increase in the number of questions tends to increase the number of reviews in the next period. Meanwhile, an increase in the number of answers appears to reduce the average content word count of reviews in the subsequent period. Our results indicate that the Q\&A system should not be simply implemented without proper planning. If the store has too many questions, especially unanswered ones, the existence of the question and answer system could actually hurt the store. On the other hand, if the store can manage to have the posted questions answered, and the depth of those answers are appropriate, then this system could be particularly beneficial to the store.

\section{Literature Review}

In this section, we review the literature that discusses the economic value of electronic commerce platforms, the economic implication of electronic word of mouth, and online discussions.

\subsection{Electronic commerce platforms}

Early IS literature in electronic commerce mostly focuses on value creation [8]. In that regard, Torkzadeh and Dhillon show that one of the key elements to create value in consumers' eyes is to build their trust [9], which has been shown to have a strong impact to consumers' satisfaction and purchase intention [10]. Several avenues are suggested in establishing the trust. For example, Pavlou and Gefen propose that using thirdparty institutional-based mechanisms (e.g., third party escrow services) could help engendering the trust in the ecommerce platform [2]. In addition, reputation systems proposed by Resnick et al. [11] has been empirically shown to have a positive impact on consumers' trust as well [12]. Furthermore, Pavlou and Dimoka argue that textual contents, in addition to numeric ratings from reputation systems, play an important role in de- veloping trust [13]. This finding is supported by Awad and Ragowsky, which find that textual contents in online review system can significant impact the trust and the effect is different across genders [3]. Given a theoretical foundation of textual contents and online reviews in building consumers trust, we then proceed to survey the literature that discusses their economic implications in the next subsection.

\subsection{Economic implication of eWOM}

Chevalier and Mayzlin are among the first studies to investigate the impact of online reviews on sales [6]. They gather book reviews and sales rank on Amazon and Barnes and Noble website to conduct difference-indifference analysis on review elements and sales. They find that review elements such as number of reviews, together with the prices, have significant impact on sales rank. In addition, Zhu and Zhang find that the impact of online reviews varies across product and consumer characteristics [7]. For example, online reviews are more influential if a product is less popular. Furthermore, Wu et al. employ the structural modeling approach to investigate the economic value of online review using a data set from a Chinese online restaurant review platform [14]. They find a significant impact of online reviews on the value of the restaurants.

Apart from the volume of the reviews, other review elements have also been shown to be significantly valuable. For one, Chintagunta et al. study the effect of online reviews on movie box office performance. They find that the valence, not the volume, seems to impact the performance of the movie in designated market area [15]. They argue that their findings can be generalized to other products that are rolled out sequentially too. Additionally, Luca empirically study the impact of online consumer reviews on Yelp.com on restaurant demand. He finds that numeric star ratings on yelp can significantly affect restaurant's revenue [16]. Furthermore, the variance of the star ratings has also been found to affect sales in certain contexts as well. Monic Sun proposes an analytical model that explains the role of the variance on sales [17]. She then uses online book review data on Amazon to empirically demonstrates that books with higher standard deviation of star ratings get better sales rank if they are unpopular (less than 4.1 star ratings). According to Lu et al. , the economic impact of online reviews is still significant in the presence of other marketing promotions (e.g., online coupon) [18].

Although there is plenty of evidence that eWOM has a significant economic impact, most of the works only investigate such an impact in a limited context. Babic et al. summarize 96 studies and find that the term "elec- 
tronic word of mouth" only refers to online reviews and social media sharing [19]. In this paper, our primary objective is to study the economic impact of eWOM in the form of online discussions that co-exist with an online review system. Hence, in the next subsection, we review literature that studies online discussions.

\subsection{Online discussions}

Online discussion forums (or online communities, electronic networks of practice) has been one of the most popular topics in IS research community in the last decade. It has been studied in many different contexts such as motivations for knowledge contribution [4], fraud detection and reporting [20], and the role of social mechanism [21]. In term of value creation, online discussion has been shown to be extremely valuable for users [22]. For example, Yan and Tan empirically show that social support in discussion groups can be beneficial to participated patients [5]. In addition, OestreicherSinger and Zalmanson use data from Last.fm to show that the increase in participation in an online community can actually increase consumers' willingness to pay for the product related to that community [23].

Regarding the economic values, Bickart and Schindler provides an early ground in the area by experimentally demonstrate that online forums are influential sources of consumer information [24]. Another stream of research that is closely related to our study is in finance where user generated contents have been shown to significantly impact stock performance [25]. To the best of our knowledge, our work is the first in providing a formal empirical analysis on the economic impact of online discussion system. In addition, the online discussion system in our study co-exists with online review systems thus enable us to compare and contrast the differences in different types of electronic word of mouth.

In sum, previous literature has extensively examined many forms of electronic word of mouth. However, studies that investigate economic implications are mostly limited to online reviews and social media content sharing. Meanwhile, previous works that study online discussions largely focus on user participation and content. To the best of our knowledge, our work is the first to empirically analyze the economic implications of online discussion systems, particularly the impact of question and answer elements on product sales.

\section{Hypotheses Development}

In this section, we describe the context of our study and develop and present our hypotheses. We focus on online discussions and reviews that involve single- purchase products that are experience goods. Examples of these products are books, music, and video games. Since observing the characteristics of these products are difficult until product consumption occurs, electronic word of mouth such as online reviews and discussions could be useful in helping potential consumers to make their purchasing decision.

Next, we develop our main hypotheses. The first variable of interest is the volume of questions per product. The volume is an important element as previous literature has shown its significant impact on product sales [6]. Therefore, as questions could be classified as a type of eWOM, we expect that the volume of questions should have a significant impact on product sales as well. However, since there are limited prior works and theoretical background that could be drawn to predict the direction of this relationship, we motivate this hypothesis as an open empirical question. On the one hand, eWOM is likely correlated with the popularity of products, and there is evidence in the literature that an increase in the volume of eWOM is associated with an increases in sales [6, 17]. Since questions in the Q\&A platform are a form of eWOM, we hypothesize that:

Hypothesis 1a An increase in the total number of questions results in higher sales.

On the other hand, questions typically signify doubts or uncertainty by nature as defined by Merriam-Webster dictionary [26]. In addition, behavioral economics literature has associated questions and concerns [27]. Furthermore, a large number of questions has been shown to impose a negative sentiment and thus deter potential participants [28]. If this effect is dominant,

Hypothesis 1b An increase in the total number of questions results in lower sales.

The second variable of interest is the volume of answers per product. In the same way as Hypothesis 1a and $1 \mathrm{~b}$, this value captures the impact of eWOM on product sales. However, since answer is defined by the Merriam-Webster dictionary as a response to the question [29], it is natural to expect that content associated with answers contribute to clarification as opposed to creating doubt in the case of questions. Therefore, we theorize that consumers perceive answers as positive sentiment. Hence,

Hypothesis 2 An increase in the total number of answers results in higher sales.

Our first two hypotheses focus on the volume of questions and answers, which represent high-level mea- 
sures of questions and answers in consumers' eyes. In the following hypotheses, we are interested to see the effect of the depth of questions and answers on product sales. If the depth of questions and answers has a significant effect, it could imply that consumers carefully consume the information in the question and answer system and pay attention to details. On the other hand, if the depth of questions and answers is not significant, then consumers merely use this system to attain some signals regarding the product. To measure the depth, we adopt an approach employed in [30] by using word count as a proxy to measure the depth of eWOM of experience goods. An online discussion setting naturally tends to create extensive engagement among users as asking and answering questions requires non-negligible effort. Therefore, we expect users to pay attention to the details of the question and answer system. Based on explanations outlined in the development of Hypothesis 1a, if the volume of questions is representative of customers' interest in the product, we anticipate the relationship of the question depth and product sales to be similar to that between the volume of questions and sales. Therefore,

Hypothesis 3a An increase in the depth of questions results in higher sales.

On the other hand, as outlined in the development of Hypothesis $1 \mathrm{~b}$, questions could convey a negative signal as they might be associated with doubt and uncertainty about the product, and thus:

Hypothesis $3 \mathbf{b}$ An increase in the depth of questions results in lower sales.

Since we expect to see the effect of the depth of questions on product sales, it is natural to expect a significant impact for the depth of answers on product sales as well. We employ the same measurement by using answer word count as a proxy for answer depth. We theorize that the depth has an effect on product sales using the same rationale discussed in the development of the previous Hypothesis. However, as mentioned in Hypothesis 2 , answers are usually associated with a positive signal in consumers' perception, so we expect the direction of the effect of answer depth on product sales to be the opposite of the effect of question depth on product sales. In other words,

Hypothesis 4 An increase in the depth of answers results in higher sales.

Our last variable of interest is the fraction of questions that have at least one answer. This variable is of interest as it represents how much of the concerns raised by questioning consumers have been addressed. Our previous hypotheses argue that questions are usually perceived as negative signals, and they thus have a negative impact on product sales. However, it is important to note that if most (or all) of the questions have some answers, this could be a signal for clarification of most of the uncertainties and doubts created by the questions. In other words, not all the questions are equal. Therefore, disregarding this variable could leave an important effect of question and answer elements out of our analysis. When consumers observe that the fraction of questions with answers increases, we expect them to perceive that more concerns regarding the product have been addressed, which implies that the perceived risk of acquiring the product is reduced. Therefore,

Hypothesis 5 An increase in the fraction of questions that have answers results in higher sales.

\section{Data Descriptions}

We collected our data from two retailers. The primary platform is Amazon.com ("Platform A" hereinafter), which offers both a review system and a question and answer system. Another platform ("Platform B") is a large retailer which offers only a review system. For product type, we choose video games as they are experience goods with a reasonably long product life cycle, so that our dataset contains both new and old products. Furthermore, previous studies have used video games to investigate the effect of online reviews on sales [7]. Also video games have a unique characteristic in that many products in this category allow pre-ordering. Hence, those products are listed before the release date. In that period, users are allowed to ask and answer questions but cannot post reviews on those products.

We employed an automated script to obtain the list of products in the video game category on both retailers. Then, for each product, the script collected product characteristics, review elements, and Q\&A elements. Also, for each product, the script acquired time-sensitive product information such as sales rank and price. We started collecting the data on a daily basis between March 1, 2015 and September 30, 2015. We then removed invalid products such as products that are incorrectly classified as a video game from our data set. In addition, we removed duplicated products such as pre-owned and digital versions of the same product. The total number of products we collected from platform $\mathrm{A}$ is 1,945 while there are 1,806 products on platform B. For those products, 891 products are matched across two platforms. On platform A, 
there are 266,376 reviews from 192,836 users (excluding 457 anonymous reviews), 15,342 questions from 11,868 users, and 32,169 answers from 24,515 users. Meanwhile, on platform B, there are 101,468 reviews from 67,088 users. The summary statistics of price, sales rank, review elements, and question and answer elements are presented in Tables 1 and 2.

\section{Table 1: Summary statistics of review and question\&answer elements on platform $\mathrm{A}$ (Cross-sectional, per product)}

\begin{tabular}{|l|l|l|l|l|}
\hline Variable & Mean & Minimum & Maximum & Std. Dev. \\
\hline Price & 21.8492 & 0.01 & 748.99 & 30.5893 \\
\hline Sales rank & $7,748.7178$ & 1 & $1,021,885$ & $22,417.1078$ \\
\hline Number of reviews & 135.8967 & 0 & 986 & 180.1284 \\
\hline Average star ratings & 4.0722 & 1 & 5 & 0.6022 \\
\hline Average content word count & 86.3714 & 2 & 709.50 & 61.1407 \\
\hline Number of questions & 7.6992 & 0 & 115 & 11.0465 \\
\hline Average question word count & 12.1548 & 3.50 & 42 & 3.9851 \\
\hline Number of answers per product & 16.1887 & 0 & 249 & 25.6136 \\
\hline Average answer word count & 22.6070 & 1 & 824 & 27.4209 \\
\hline
\end{tabular}

Table 2: Summary statistics of review elements on platform B (Cross-sectional, per product)

\begin{tabular}{|l|l|l|l|l|}
\hline Variable & Mean & Minimum & Maximum & Std. Dev. \\
\hline Price & 25.9575 & 0.99 & 399.99 & 24.4664 \\
\hline Sales rank & $127,127.4171$ & 20 & 400,606 & $129,258.2978$ \\
\hline Number of reviews & 56.0725 & 0 & 980 & 132.3278 \\
\hline Average star ratings & 4.2347 & 1 & 5 & 0.6417 \\
\hline Average content word count & 37.5335 & 10 & 254 & 21.7141 \\
\hline
\end{tabular}

\section{Model Specifications}

In this section, we describe our empirical modeling approach. Our primary dependent variable is the natural logarithm of sales rank. It is a proxy to the sales volume of the products, which is an ideal dependent variable but not available to us. Previous studies have shown that the relationship between log sales rank and log sales is close to linear [31,32]. It is also widely used in previous works to measure the effect of online reviews on product sales $[6,17]$.

To measure the effect of question and answer elements on sales, we first have to consider the potential issue of endogeneity and simultaneity. Some product characteristics are typically unobservable to researchers but could be correlated with our dependent variable (i.e., product sales). In this paper, we adopt the difference-indifferences approach, which has been used extensively to unveil the effect of online review elements on product sales $[6,7,17]$. It is also widely adopted to establish causal arguments in other contexts when endogeneity issues are present $[33,34]$. In addition, we also include product popularity as an interaction term in our model since prior works have shown its moderating effect on the impact of eWOM volume on sales [7].
Consider product $i$ that is available on both platforms. Its sales is a function of a productplatform fixed effect, a product fixed effect, a price $\left(\right.$ Price $\left._{i}\right)$, and review elements (ReviewElement $s_{i, t-1}$ ) consisting of the lagged average star ratings and log total number of reviews, the lagged average review word count (AvgReviewLength $\left.h_{i, t-1}\right)$, lagged total number of questions (NumQuestion ${ }_{i, t-1}$ ), lagged total number of answers (NumAnswer ${ }_{i, t-1}$ ), lagged average word count of questions in the previous period (AvgQuestionLength $h_{i, t-1}$ ), the lagged average answer word count (AvgAnswerLength $h_{i, t-1}$ ), and the lagged fraction of questions with answers (FracQuestionWithAnswer ${ }_{i, t-1}$ ). Note that question and answer elements only exist on platform A. Next, we adopt a specification similar to that used in [6] by allowing prices and review elements from one platform to affect sales on another platform. Also, we follow [7] by including product popularity as an interaction term. We define product $i$ to be popular at time $t$ if its sales rank on Amazon at that time is lower than 1,000. We also vary this definition in robustness tests and find that our results are robust. Therefore, the dummy variable popular $_{i, t}$ indicates if a product is popular. We eliminate the product fixed effect by differencing the data across platforms and eliminate the product-platform fixed effect by differencing the data across time. Our final specification is:

$$
\begin{aligned}
& \Delta \Delta \ln \left(\text { SalesRank }_{i, t}\right)=\beta_{1} \Delta \ln \left(\text { Price }_{i, t}^{A}\right)+\beta_{2} \Delta \ln \left(\text { Price }_{i, t}^{B}\right) \\
& +\beta_{3} \Delta\left(\text { ReviewElement } s_{i, t-1}^{A}\right)+\beta_{4} \Delta\left(\text { ReviewElement }_{i, t-1}^{B}\right) \\
& +\beta_{7} \Delta \ln \left(\text { NumQuestion }_{i, t-1}^{A}\right)+\beta_{8} \Delta \ln \left(\text { NumAnswer }_{i, t-1}^{A}\right) \\
& \left.+\beta_{9} \Delta \text { AvgQuestionLength } h_{i, t-1}^{A}\right) \\
& \left.+\beta_{10} \Delta A v g \text { AnswerLengt } h_{i, t-1}^{A}\right) \\
& +\beta_{1} 1 \Delta\left(\text { FracQuestionWithAnswer }{ }_{i, t-1}^{A}\right) \\
& +\beta_{12} \Delta\left(\ln \left(\text { Price }_{i, t}^{A}\right) \times \text { popular }_{i, t}\right) \\
& +\beta_{13} \Delta\left(\ln \left(\text { Price }_{i, t}^{B}\right) \times \text { popular }_{i, t}\right) \\
& +\beta_{14} \Delta\left(\text { ReviewElements } s_{i, t-1}^{A} \times \text { popular }_{i, t}\right) \\
& +\beta_{15} \Delta\left(\text { ReviewElement } s_{i, t-1}^{B} \times \text { popular }_{i, t}\right) \\
& +\beta_{16} \Delta\left(\text { NumQuestion }_{i, t-1}^{A} \times \text { popular }_{i, t}\right) \\
& +\beta_{17} \Delta\left(\text { NumAnswer }_{i, t-1}^{A} \times \text { popular }_{i, t}\right)+\varepsilon_{i, t}
\end{aligned}
$$

Based on this specification, we constructed our data into a panel such that each observation corresponds to a product, and each time period is a calendar month. Note that although we have 7 months of data in total, we lose one month in the lagged independent variables construction and another month when differencing the data across time. Therefore, our final panel dataset consists of 891 products and 5 time periods. 


\section{Empirical Results}

\subsection{The Effect of Q\&A Elements on Sales}

In this subsection, we present results from our empirical analysis specified in Section 5. We begin our analysis by testing our outcome variable for serial correlations using the package XTSERIAL in STATA [35]. We do not find evidence that our dependent variable has a significant first-order serial correlation (F-value 0.033 with $\mathrm{p}$-value 0.8549 ). Therefore, we employ the standard panel data model in our analysis. Table 3 presents the regression results of the differencein-differences specification. In Model 1, we include only price and review elements. In Model 2, we add question and answer elements to the model but leave the interaction terms out. In Model 3, we add the interaction terms to the model. Recall that our dependent variable is the difference of differences sales ranks on both platforms (i.e., $\left(\ln \left(\operatorname{rank}_{i, t+1}^{A}\right)-\ln \left(\operatorname{rank}_{i, t+1}^{B}\right)\right)-$ $\left.\left(\ln \left(\operatorname{rank}_{i, t}^{A}\right)-\ln \left(\operatorname{rank}_{i, t}^{B}\right)\right)\right)$. Therefore, when the effect of sales is positive (i.e., product sales increases), our dependent variable will decrease (as the sales rank decreases).

We find that product prices indeed significantly affect sales. When product price on platform A increases, the relative sales (the difference between product sales on platforms A and B) decreases. Intuitively, when product price on platform $B$ increases, the relative sales of that product increases. These results imply that consumers compare product prices across platforms. Interestingly, on average, the effect of platform B's price to product sales, the "cross-platform effect", is much stronger than the effect of the price on platform $\mathrm{A}$, the "direct effect". In addition, when we separate products into popular and less popular products, we find that the effect of price on both platforms on the relative sales is similar for popular products. Meanwhile, the price on platform $B$ has a stronger effect on the relative sales for less popular ones. As for review elements, we discover that the effects of review elements on product sales are not particularly strong. First, the direct effect of average star ratings is insignificant. However, the cross-platform effect is negative and significant for less popular products. Second, for popular products, the direct effect of the total number of reviews on sales is positive while the cross-platform effect of the total number of reviews on sales is negative. However, these effects flip to an opposite direction for less popular products. Third, both the direct and cross-platform effects of review depth on sales are insignificant.

Next, we find that the effects of question and answer elements on product sales mostly align with our hypotheses. First, the effect of total questions on sales is negative as theorized. However, it is not statistically significant for products on average and for less popular products. The effect is only significant for popular products. Second, the direction of the effect of total answers on sales is positive, which is also in line with our hypothesis. However, this effect is not statistically significant in any of the cases. Interestingly, the effect of total answers for less popular products is much stronger than that for popular products, but it is also not statistically significant. Third, an increase in average question depth has a negative effect on sales, hence Hypothesis $3 \mathrm{~b}$ is supported. Also, Hypothesis 4 is supported as we find that an increase in average answer depth has a positive effect on sales. Furthermore, an increase in the fraction of questions with answers has a positive impact on sales. Therefore, Hypothesis 5 is also statistically supported.

Our findings regarding the effect of question and answer elements are particularly interesting and insightful. We find that the Q\&A system has a potential economic impact on online shopping platforms. However, such an impact is mixed as question elements appear to have a negative impact on sales while answer elements tend to have a positive impact. Furthermore, even though the number of questions negatively affects sales, an increase in the fraction of questions with at least one answer actually affect sales in a positive direction. Therefore, it is essentially the number of unanswered questions that really hurts the platform. Additionally, users seem to pay attention to details rather than relying only on the overall statistics such as number of questions and answers. We observe that both the average depth of questions and answers affect sales significantly while the total number of questions and answers do not, except for the case of total number of questions for popular products. However, summary statistics do play a role when it comes to the fraction of questions with answers as its increase could positively affect sales. Therefore, implementing the question and answer system on an online shopping platform must be carefully planned and executed as its effect is two-fold. If the system has many questions but a relatively low number of answers, then the presence of the Q\&A system could actually hurt the bottom line of the platform. However, if the system is implemented appropriately (e.g., there are a wide-range of answers and most of the questions are answered) then the economic impact of the system could be positively significant as intended. With the Q\&A system, platform managers should make sure that the technical environment supports user interaction with the system, especially for answering questions. In the same way, there should be mechanisms in place to encourage users to answer questions, especially questions without an answer. 
Table 3: The effect of question and answer elements on sales

\begin{tabular}{|c|c|c|c|}
\hline & (1) & (2) & (3) \\
\hline$\Delta \ln ($ price $)(\mathrm{A})$ & $.0869 * * *(.0338)$ & $.2004 * * *(.0492)$ & $.1461 * * *(.0512)$ \\
\hline$\Delta \ln ($ price $)(\mathrm{B})$ & $-.4697 * * *(.1218)$ & $-.7116 * * *(.1041)$ & $-.5617 * * *(.1340)$ \\
\hline$\Delta$ average star ratings $(\mathrm{A})$ & $-.0561(.1766)$ & $.1084(.1827)$ & $.0518(.2217)$ \\
\hline$\Delta$ average star ratings (B) & $.2250(.1808)$ & $.3853 *(.2011)$ & $.4453 * *(.2177)$ \\
\hline$\Delta \ln ($ total no. of review) (A) & $.2725(.1720)$ & $-.0550(.1750)$ & $.4315^{*}(.2351)$ \\
\hline$\Delta \ln ($ total no. of review) (B) & $-.2154 *(.1141)$ & $-.1845^{*}(.1061)$ & $-.4761 * * *(.1709)$ \\
\hline$\Delta$ avg. review word count (A) & $.0004(.0009)$ & $-.0004(.0010)$ & $.0001(.0011)$ \\
\hline$\Delta$ avg. review word count (B) & $-.0004(.0037)$ & $-.0041(.0054)$ & $-.0033(.0057)$ \\
\hline$\Delta \ln$ (total no. of question) (A) & & $.1880(.1543)$ & $.0892(.1807)$ \\
\hline$\Delta$ avg. question word count (A) & & $.0305 * *(.0140)$ & $.0254 *(.0141)$ \\
\hline$\Delta \ln ($ total no. of answer) (A) & & $-.1134(.1199)$ & $-.1425(.1442)$ \\
\hline$\Delta$ avg. answer word count (A) & & $-.0116 * * *(.0038)$ & $-.0132 * * *(.0039)$ \\
\hline$\Delta$ fraction of question with answer (A) & & $-.7970 * *(.3840)$ & $-.7243 *(.4005)$ \\
\hline$\Delta \ln ($ price $) \times$ popular $(\mathrm{A})$ & $.5967 * * *(.1671)$ & & $.5136 * * *(.1725)$ \\
\hline$\Delta \ln ($ price $) \times$ popular $(\mathrm{B})$ & $-.4703 * *(.2021)$ & & $-.3938 *(.2104)$ \\
\hline$\Delta$ avg. star ratings $\times$ popular $(\mathrm{A})$ & $-.0372(.3412)$ & & $.0949(.4021)$ \\
\hline$\Delta$ avg. star ratings $\times$ popular $(\mathrm{B})$ & $-.0618(.4773)$ & & $-.1677(.5694)$ \\
\hline$\Delta \ln ($ total no. of review) $\times$ popular $(\mathrm{A})$ & $-.3990 *(.2076)$ & & $-.9467 * * *(.2706)$ \\
\hline$\Delta \ln ($ total no. of review $) \times$ popular $(B)$ & $.1361(.1475)$ & & $.5162 * *(.2021)$ \\
\hline$\Delta \ln ($ total no. of question $) \times$ popular $(\mathrm{A})$ & & & $.8028 *(.3536)$ \\
\hline$\Delta \ln ($ total no. of answer $) \times$ popular $(\mathrm{A})$ & & & $-.0090(.2031)$ \\
\hline Intercept & $-.0188(.0126)$ & $-.0236^{*}(.0129)$ & $-.0249(.0144)$ \\
\hline Observations & 2,176 & 1,893 & 1,893 \\
\hline$R^{2}$ & 0.0372 & 0.0467 & 0.0591 \\
\hline
\end{tabular}

\subsection{The Effect of Q\&A Elements on Initial Sales}

In this subsection, we extend our analysis by investigating the effect of question and answer elements on initial sales. It is worth highlighting that unlike reviews, discussions regarding a product can occur before the product release date as questions can be asked and answered even before that product becomes available. Hence, we direct our focus of this subsection to the effect of question and answer elements on product sales in the first day of product release. The specification for this analysis is as follows:

$$
\begin{aligned}
& \Delta \ln \left(\text { SalesRank }_{i}\right)=\beta_{0}+\beta_{1} \Delta \ln \left(\text { Price }_{i}\right) \\
& +\beta_{2}\left(\text { NumQuestion }_{i}\right)+\beta_{3}\left(\text { NumAnswer }_{i}\right) \\
& +\beta_{4}\left(\text { AvgQuestionLength }_{i}\right)+\beta_{5}\left(\text { AvgAnswerLength }_{i}\right) \\
& +\beta_{6}\left(\text { FracQuestionWithAnswer }_{i}\right)+\varepsilon_{i}
\end{aligned}
$$

where $\Delta$ SalesRank $_{i}$ is the difference in sales rank of product $i$ between two platforms on the first day of product release, $\Delta \ln \left(\right.$ Price $\left._{i}\right)$ is the difference in log price of product $i$ between platforms, NumQuestion ${ }_{i}$ and NumAnswer ${ }_{i}$ are the number of questions and answers product $i$ has on Amazon prior to the release date, AvgQuestionLength ${ }_{i}$ and AvgAnswerLength ${ }_{i}$ are the average word count of those questions and answers, and FracQuestionWithAnswer ${ }_{i}$ is the total number of questions that have at least one answer.

Our dataset contains products that are released between March and September 2015. Although there are 109 products in total, 12 of them do not have any questions posted on Amazon before the release date. Also, only 59 products have at least one question on Amazon and positive initial sales rank on both platforms.

The results of our analysis are presented in Table 4. Interestingly, we find that the number of questions posted before the release date positively correlates with lower sales rank (i.e., higher sales) although the significant level is only marginal. However, the correlation between the fraction of questions with answers and the sales rank is particularly strong. That is, the more questions with at least one answer posted before the product release date, the product attains higher initial sales. 
Table 4: The effect of question and answer elements on initial sales

\begin{tabular}{ll}
\hline & $\Delta \ln \left(\right.$ SalesRank $\left._{i}\right)$ \\
\hline$\Delta \ln ($ Price $)$ & $-3.4377(2.8411)$ \\
NumQuestion & $-0.0144 *(0.0079)$ \\
NumAnswer & $0.0183(0.0259)$ \\
AvgQuestionLength & $0.0191(0.0344)$ \\
AvgAnswerLength & $0.0008(0.0011)$ \\
FracQuestionWithAnswer & $-1.1116^{* * *}(0.4012)$ \\
Intercept & $-4.1487 * *(0.5181)$ \\
& \\
Observation & 59 \\
$R^{2}$ & 0.2550 \\
\hline$* p<0.10 ; * * p<0.05 ; * * * p<0.01$
\end{tabular}

\subsection{The Effect of Question and Answer Ele- ments on Reviews}

In this subsection, we shift our focus to examine the relationship between the Q\&A system and the traditional online review system. We reconstruct our dataset to be a panel dataset at a product level with monthly observations between March and September 2015. However, we instead use the total number of reviews and average review word count as dependent variables in this analysis while having question and answer elements of interest as independent variables of the model. Our specifications are as follows:

$$
\begin{aligned}
& \Delta \Delta \ln \left(\text { NumReview }_{i, t}\right)=\beta_{0}+\beta_{1} \Delta \ln \left(\text { NumQuestion }_{i, t-1}\right) \\
&+\beta_{2} \Delta \ln \left(\text { NumAnswer }_{i, t-1}\right) \\
&\left.+\beta_{3} \Delta \text { AvgQuestionLength } h_{i, t-1}\right) \\
&+\beta_{4} \Delta \text { AvgAnswerLength } \\
&i, t-1) \\
& \\
&+\beta_{5} \text { MonthSinceRelease }_{i}+\varepsilon_{i, t}
\end{aligned}
$$

$$
\begin{aligned}
& \Delta \Delta \text { AvgReviewLengt }_{i, t}=\beta_{0}+\beta_{1} \Delta \ln \left(\text { NumQuestion }_{i, t-1}\right) \\
&+\beta_{2} \Delta \ln (\text { NumAnswer } \\
&i, t-1) \\
& \\
&\left.+\beta_{3} \Delta \text { AvgQuestionLength } h_{i, t-1}\right) \\
&\left.+\beta_{4} \Delta \text { AvgAnswerLength }{ }_{i, t-1}\right) \\
&+\beta_{5} \text { MonthSinceRelease }_{i}+\varepsilon_{i, t}
\end{aligned}
$$

In above equations, $\Delta \Delta \ln \left(\right.$ NumReview $\left._{i, t}\right)$ is the difference between the log total number of reviews across platforms and time for product $i$, $\Delta \Delta$ AvgReviewLengt $h_{i, t}$ is the difference between the average review word count for product $i$ across platforms and time, NumQuestion ${ }_{i, t-1}$, NumAnswer i $_{i-1}$, AvgQuestionLengt $h_{i, t-1}$, and AvgAnswerLengt $h_{i, t-1}$ are all lagged variables. MonthSinceRelease ${ }_{i}$ is the number of months since the release date of product $i$.
Table 5 and 6 show the results of our analysis regarding the effect of question and answer elements on review elements. We find that an increase in the number of questions in the previous period seems to positively affect the number of reviews in the following period. In other words, higher number of questions regarding a product leads reviewers to write more review about that product. Meanwhile, an increase in the number of answers in the previous period appears to negatively influence review word counts in the following period. Our findings yield interesting insights into the interaction between the question and answer system and the review system. The questions could stimulate users in the platform to share their experience on the review systems more often. However, as questions attract more answers, the following reviews tend to be shorter. Therefore, managers should exercise cautions when implementing both question and answer system and review system together as the Q\&A system could play both supporting and cannibalizing role on the online review system.

Table 5: The effect of question and answer elements on total number of reviews

\begin{tabular}{ll}
\hline & $\Delta \Delta \ln ($ NumReview $)$ \\
\hline$\Delta \ln$ (NumQuestion) & $1.4634 * *(0.6712)$ \\
$\Delta \ln$ (NumAnswer) & $0.4586(0.4274)$ \\
$\Delta$ AvgQuestionLength & $0.0411(0.0758)$ \\
$\Delta$ AvgAnswerLength & $-0.0023(0.0049)$ \\
MonthSinceRelease & $-0.0117 * * *(0.0029)$ \\
Intercept & $-0.3368 * *(0.0835)$ \\
& \\
Observations & 3720 \\
$R^{2}$ & 0.0317 \\
\hline$* p<0.10 ; * * p<0.05 ; * * * p<0.01$
\end{tabular}

Table 6: The effect of question and answer elements on review word count

\begin{tabular}{ll}
\hline & $\Delta \Delta$ AvgReviewLength \\
\hline$\Delta \ln ($ NumQuestion) & $-2.7461(3.4000)$ \\
$\Delta \ln$ (NumAnswer) & $-6.2972 * * *(2.2240)$ \\
$\Delta$ AvgQuestionLength & $-0.0711(0.3552)$ \\
$\Delta$ AvgAnswerLength & $0.1206(0.0951)$ \\
MonthSinceRelease & $0.0827 * * *(0.0181)$ \\
Intercept & $-3.6590 * *(0.5515)$ \\
& \\
Observations & 2467 \\
$R^{2}$ & 0.0100 \\
\hline$* p<0.10 ; * * p<0.05 ; * * *<0.01$
\end{tabular}




\section{Discussions and Conclusions}

In recent years, many websites and online marketplaces have started to offer a question and answer system that allows users to ask and answer questions regarding available products and services. At first glance, it appears to be a good addition to the platform as it can be considered another form of electronic word of mouth, which could positively impact product sales. However, it is also possible that the question and answer system could cannibalize the online review system and thus eventually impact sales in a negative direction. Since there is a limited prior works that examine the economic implications of an online discussion systems, we are motivated to study this problem. We collect data from two online marketplaces where one offers both Q\&A and online review systems while another one offers only an online review system. Following that, we leverage the difference-in-differences approach to investigate the effect of question and answer elements on product sales.

Our analysis finds that question and answer elements indeed influence product sales. Question elements appear to negatively impact sales while answer elements affect sales positively. Consumers in an early purchase cycle seem to direct their focus to "summary variables" such as number of questions and the fraction of questions with answers. However, in the longer run, customers tend to pay attention to more details, particularly the depth of those discussions instead. Lastly, we examine the influence of question and answer elements on reviews. Interestingly, an increase in the number of questions positively affect the number of reviews in the following period while an increase in the number of answers decreases review word count in the next period.

Our study yields relevant and insightful managerial implications. We show that the Q\&A system must be managed appropriately to attain the intended results. For instance, the platform could be better off without the Q\&A system if it attracts questions but fails to attain answers. We also demonstrate that the number of questions and answers are not as important as their depth regarding their effect on product sales. Hence, managers have to be careful in constructing incentive policies to attract participants. Our work also contributes to the body of literature in economics of IS, specifically in the area of online discussion and electronic commerce. We are the first to offer empirical evidence of the economic values of the online discussion feature. In addition, we also empirically study the interaction between question and answer elements and online review elements.

Finally, we conclude our work by discussing some limitations and potential avenue for future research. Our work use sales rank as the main dependent variable as it is available to the public. Although previous literature has established that sales rank tend to have a linear relationship with sales, it would be ideal to have sales as the main dependent variable. Second, our work focuses only on the implication of question and answer elements on the sales of experience goods. One potential extension would be to study if our results hold for search goods and identify if any specific product characteristics could moderate the influence of question and answer elements. Third, although our analysis does not include textual content analysis of the questions and answers as they tend to be very brief, an interesting extension would be to study how question and answer elements influence the textual content of product reviews. Lastly, another valuable addition to our paper would be to study the behavioral side of the question and answer system. Particularly, studies that develop consumer behavior theories on how consumers adopt questions and answers into their purchase decision process.

\section{References}

[1] D. Gefen, E. Karahanna, and D. W. Straub, "Trust and tam in online shopping: an integrated model," MIS quarterly, vol. 27, no. 1, pp. 51-90, 2003.

[2] P. A. Pavlou and D. Gefen, "Building effective online marketplaces with institution-based trust," Information systems research, vol. 15, no. 1, pp. 3759, 2004.

[3] N. F. Awad and A. Ragowsky, "Establishing trust in electronic commerce through online word of mouth: An examination across genders," Journal of Management Information Systems, vol. 24, no. 4, pp. 101-121, 2008.

[4] M. M. Wasko and S. Faraj, "Why should I share? examining social capital and knowledge contribution in electronic networks of practice," MIS quarterly, pp. 35-57, 2005.

[5] L. Yan and Y. Tan, "Feeling blue? go online: An empirical study of social support among patients," Information Systems Research, vol. 25, no. 4, pp. 690-709, 2014.

[6] J. A. Chevalier and D. Mayzlin, "The effect of word of mouth on sales: Online book reviews," Journal of marketing research, vol. 43, no. 3, pp. 345-354, 2006.

[7] F. Zhu and X. Zhang, "Impact of online consumer reviews on sales: The moderating role of product and consumer characteristics," Journal of marketing, vol. 74, no. 2, pp. 133-148, 2010.

[8] R. L. Keeney, "The value of internet commerce to the customer," Management science, vol. 45, no. 4, pp. 533-542, 1999. 
[9] G. Torkzadeh and G. Dhillon, "Measuring factors that influence the success of internet commerce," Information Systems Research, vol. 13, no. 2, pp. 187-204, 2002.

[10] Y. Fang, I. Qureshi, H. Sun, P. McCole, E. Ramsey, and K. H. Lim, "Trust, satisfaction, and online repurchase intention: the moderating role of perceived effectiveness of e-commerce institutional mechanisms," Mis Quarterly, vol. 38, no. 2, pp. 407-427, 2014.

[11] P. Resnick, K. Kuwabara, R. Zeckhauser, and E. Friedman, "Reputation systems," Communications of the ACM, vol. 43, no. 12, pp. 45-48, 2000.

[12] M. I. Melnik and J. Alm, "Does a seller's ecommerce reputation matter? evidence from ebay auctions," Journal of Industrial Economics, pp. 337349, 2002.

[13] P. A. Pavlou and A. Dimoka, "The nature and role of feedback text comments in online marketplaces: Implications for trust building, price premiums, and seller differentiation," Information Systems Research, vol. 17, no. 4, pp. 392-414, 2006.

[14] C. Wu, H. Che, T. Y. Chan, and X. Lu, "The economic value of online reviews," Marketing Science, vol. 34, no. 5, pp. 739-754, 2015.

[15] P. K. Chintagunta, S. Gopinath, and S. Venkataraman, "The effects of online user reviews on movie box office performance: Accounting for sequential rollout and aggregation across local markets," Marketing Science, vol. 29, no. 5, pp. 944-957, 2010.

[16] M. Luca, "Reviews, reputation, and revenue: The case of yelp. com," tech. rep., Harvard Business School, 2011.

[17] M. Sun, "How does the variance of product ratings matter?," Management Science, vol. 58, no. 4, pp. 696-707, 2012.

[18] X. Lu, S. Ba, L. Huang, and Y. Feng, "Promotional marketing or word-of-mouth? evidence from online restaurant reviews," Information Systems Research, vol. 24, no. 3, pp. 596-612, 2013.

[19] A. Babic, F. Sotgiu, K. de Valck, and T. H. Bijmolt, "The effect of electronic word of mouth on sales: A meta-analytic review of platform, product, and metric factors," Journal of Marketing Research, forthcoming, 2015.

[20] C. E. H. Chua, J. Wareham, and D. Robey, "The role of online trading communities in managing internet auction fraud," MIS Quarterly, pp. 759-781, 2007.

[21] S. L. Johnson, S. Faraj, and S. Kudaravalli, "Emergence of power laws in online communities: the role of social mechanisms and preferential attach- ment," Mis Quarterly, vol. 38, no. 3, pp. 795-808, 2014.

[22] A. Armstrong and J. Hagel, "The real value of online communities," Knowledge and communities, pp. 85-95, 2000.

[23] G. Oestreicher-Singer and L. Zalmanson, "Content or community? a digital business strategy for content providers in the social age," MIS Quarterly, vol. 37, no. 2, 2013.

[24] B. Bickart and R. M. Schindler, "Internet forums as influential sources of consumer information," Journal of interactive marketing, vol. 15, no. 3, pp. 31-40, 2001.

[25] S. Tirunillai and G. J. Tellis, "Does chatter really matter? dynamics of user-generated content and stock performance," Marketing Science, vol. 31, no. 2, pp. 198-215, 2012.

[26] Question, Merriam-Webster.com. MerriamWebster, 2016.

[27] S. J. Solnick and D. Hemenway, "Is more always better?: A survey on positional concerns," Journal of Economic Behavior \& Organization, vol. 37, no. 3, pp. 373-383, 1998.

[28] E.-O. Im and W. Chee, "Issues in an internet survey among midlife asian women," Health Care for Women International, vol. 25, no. 2, pp. 150-164, 2004.

[29] Answer, Merriam-Webster.com. MerriamWebster, 2016.

[30] S. M. Mudambi and D. Schuff, "What makes a helpful review? a study of customer reviews on amazon. com," MIS quarterly, vol. 34, no. 1, pp. 185-200, 2010.

[31] E. Brynjolfsson, Y. Hu, and M. D. Smith, "Consumer surplus in the digital economy: Estimating the value of increased product variety at online booksellers," Management Science, vol. 49, no. 11, pp. 1580-1596, 2003.

[32] M. Schnapp and T. Allwine, "Mining of book data from amazon. com," in Presentation at the UCB/SIMS web mining conference, 2001.

[33] K. Hosanagar, D. Fleder, D. Lee, and A. Buja, "Will the global village fracture into tribes? recommender systems and their effects on consumer fragmentation," Management Science, vol. 60, no. 4, pp. 805-823, 2013.

[34] J. Chan and A. Ghose, "Internets dirty secret: assessing the impact of online intermediaries on hiv transmission," MIS Quarterly, vol. 38, no. 4, pp. 955-976, 2014.

[35] D. M. Drukker et al., "Testing for serial correlation in linear panel-data models," Stata Journal, vol. 3, no. 2, pp. 168-177, 2003. 